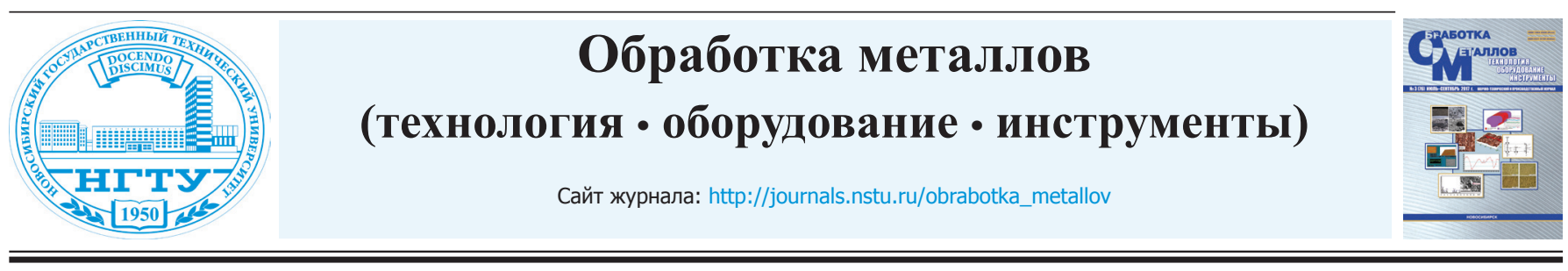

Структурные особенности плазменных покрытий $\mathrm{B}_{4} \mathrm{C}-\mathrm{Ni}-\mathrm{P}$

Елена Корниенко ${ }^{1, a, *}$, Виктор Кузьмин ${ }^{2, b}$, Василий Ложкин ${ }^{1, c}$, Игорь Гуляев $^{2, d}$,
Александр Сивков ${ }^{3, e}$, Александр Ивашутенко ${ }^{3, f}$, Ильяс Рахматуллин ${ }^{3, g}$,
Дмитрий Сергачев ${ }^{2, h}$, Виктория Безрукова

${ }^{1}$ Новосибирский государственный технический университет, пр. К. Маркса, 20, г. Новосибирск, 630073, Россия

${ }^{2}$ Институт теоретической и прикладной механики им. С.А. Христиановича СО РАН, ул. Институтская, 4/1, г. Новосибирск, 630090, Россия

${ }^{3}$ Национальный исследовательский Томский политехнический университет, пр. Ленина, 30, г. Томск, 634050, Россия

${ }^{a}$ (iD http://orcid.org/0000-0002-5874-5422, @ kornienko_ee@mail.ru, ${ }^{b}$ iD http://orcid.org/0000-0002-9951-7821, @ vikuzmin57@mail.ru,

${ }^{c}$ (D) http://orcid.org/0000-0002-1686-0369, @ lozhkin@corp.nstu.ru, ${ }^{d}$ (D) http://orcid.org/0000-0001-5186-6793, @ gulyaev@itam.nsc.ru,

${ }^{e}$ (D) http://orcid.org/0000-0002-7685-5502, @ SivkovAA@mail.ru, ${ }^{f}$ iD http://orcid.org/0000-0002-6513-5438, @ ivaschutenko@mail.ru,

$g$ (iD http://orcid.org/0000-0001-5643-2134, @ riam@tpu.ru, ${ }^{h}$ iD http://orcid.org/0000-0003-2469-5946, @ yosergach@gmail.com,

$i$ (D) http://orcid.org/0000-0002-0329-9920, @ vikabezrukova1995@mail.ru

ИНФОРМАЦИЯ О СТАТЬЕ

УДК 621.793 .71

История статьи:

Поступила: 28 июня 2017

Рецензирование: 28 июля 2017

Принята к печати: 15 августа 2017

Доступно онлайн: 15 сентября 2017

Ключевые слова:

Плазменное напыление

Карбид бора

$\mathrm{B}_{4} \mathrm{C}-\mathrm{Ni}-\mathrm{P}$

Покрытие

\section{АННОТАЦИЯ}

Рассматривается нанесение покрытия из порошка карбида бора (B C), плакированного 30 мас. \% Ni-P, на трубы из низкоуглеродистой стали при помощи воздушно-плазменного напыления с узлом кольцевого ввода. Структурные исследования покрытий проводили с использованием оптической микроскопии, растровой и просвечивающей электронной микроскопии, а также рентгенофазового анализа. Показано, что материал плазменных покрытий состоит из частиц В С размером до 30 мкм, равномерно распределенных в металлической матрице. Частицы карбида бора окружены областями с округлыми включениями размерами до 500 нм. Металлическая матрица представляет собой бориды никеля $\left(\mathrm{Ni}_{3} \mathrm{~B}, \mathrm{NiB}\right.$ и $\left.\mathrm{Ni}_{3} \mathrm{~B}_{4}\right)$. Кроме того, в материале покрытий были зафиксированы оксиды никеля и бора. Просвечивающей электронной микроскопией было выявлено, что между частицами карбида бора и металлической матрицей формируются тонкие прослойки, имеющие аморфно-кристаллическое строение. Металлическая матрица представляет собой участки, расположенные в объеме покрытий случайным образом, с нанокристаллической структурой и столбчатыми кристаллами.

Благодарности:

Работа выполнена в рамках программы повышения конкурентоспособности ТПУ среди ведущих мировых исследовательских центров.

Для цитирования: Структурные особенности плазменных покрытий В 4 -Ni-P / Е.Е. Корниенко, В.И. Кузьмин, В.С. Ложкин, И.П. Гуляев, А.А. Сивков, А.С. Ивашутенко, И.А. Рахматуллин, Д.В. Сергачев, В.А. Безрукова // Обработка металлов (технология, оборудование, инструменты). - 2017. - № 3 (76). - С. 42-50. - doi: 10.17212/1994-6309-2017-3-42-50.

\section{Введение}

Известно, что карбид бора $\left(\mathrm{B}_{4} \mathrm{C}\right)$ обладает высокой твердостью, износостойкостью и коррозионной стойкостью как при комнатных, так и при

\footnotetext{
*Адрес для переписки

Корниенко Елена Евгеньевна, к.т.н., доцент

Новосибирский государственный технический университет

пр. К. Маркса, 20, 630073, г. Новосибирск, Россия

Тел.: 8 (383) 346-11-71, e-mail: kornienko_ee@mail.ru
}

повышенных температурах [1-3]. Кроме того, он характеризуется высокой температурой плавления и низкой плотностью [2]. Это обусловливает применение $\mathrm{B}_{4} \mathrm{C}$ в качестве абразива для шлифования и полирования твердых материалов, сопел пескоструйных аппаратов и распылителей, вставок матриц пресс-форм для прессования порошков абразивных материалов, деталей насосов для перекачки холодных и нагретых растворов 
кислот, насадок сопел для распыления химически активных растворов, деталей мешалок и т. д. $[3,4]$.

Обычно из карбида бора формируют небольшие по толщине покрытия на уже готовых изделиях. В качестве основных методов нанесения покрытий из этого материала можно отметить технологии напыления [3, 5-11]. К сожалению, при нанесении покрытий из $\mathrm{B}_{4} \mathrm{C}$ возникают определенные трудности, что связано со свойствами наносимого материала. Из-за низкой теплопроводности невозможно полностью прогреть частицы порошка, а высокая твердость не позволяет частицам карбида бора пластически деформироваться на подложке или уже затвердевшем материале покрытия.

Решением данной проблемы является добавка связующего: различных металлов или химических соединений, обладающих более высокой пластичностью и теплопроводностью. Стоит отметить, что на сегодняшний день изучены структура и свойства композиционных покрытий, сформированных из смесей порошков $\mathrm{B}_{4} \mathrm{C}+\mathrm{Ni}[12,13], \mathrm{B}_{4} \mathrm{C}+\mathrm{Ti}[14], \mathrm{B}_{4} \mathrm{C}+\mathrm{Al}-\mathrm{Ti}-\mathrm{V}$ [10], $\mathrm{B}_{4} \mathrm{C}+\mathrm{Al}-\mathrm{Si}[5], \mathrm{B}_{4} \mathrm{C}+\mathrm{W}$ [14], $\mathrm{B}_{4} \mathrm{C}+\mathrm{Mo}$ [15], $\mathrm{B}_{4} \mathrm{C}+\mathrm{TiB} 2+\mathrm{TiC}[16], \mathrm{B}_{4} \mathrm{C}+\mathrm{Ni}+\mathrm{P}$ [17], полученных различными методами. Ранее авторами настоящей работы было показано, что эффективным технологическим процессом, позволяющим получать высококачественные покрытия, является воздушно-плазменное напыление с узлом кольцевого ввода порошка [18-20]. Особенности формирования композиционных покрытий $\mathrm{B}_{4} \mathrm{C}$ $\mathrm{Ni}-\mathrm{P}$ с использованием этой технологии в литературе не описаны. Таким образом, цель данной работы состоит в изучении структуры покрытий из $\mathrm{B}_{4} \mathrm{C}-\mathrm{Ni}-\mathrm{P}$, полученных методом воздушноплазменного напыления с узлом кольцевого порошка.

\section{Материалы и методы исследований}

Коммерческий порошок карбида бора $\left(\mathrm{B}_{4} \mathrm{C}\right)$, плакированный 30 вес. \% Ni-P (НПО «ТулаЧермет»), использовали в качестве материала для нанесения покрытий. Изображение частиц напыляемого порошка представлено на рис. 1. Видно, что Ni-Р-покрытие равномерно распределено на частицах произвольной формы. Размеры частиц порошка находились в диапазоне 40...100 мкм.

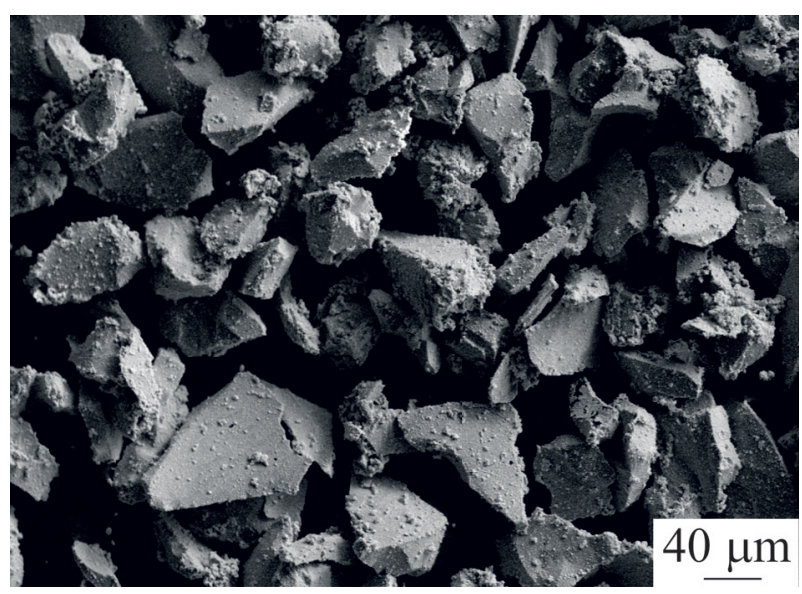

Puc. 1. Внешний вид частиц порошка $\mathrm{B}_{4} \mathrm{C}-\mathrm{Ni}-\mathrm{P}$

Fig. 1. The particles of $\mathrm{B}_{4} \mathrm{C}-\mathrm{Ni}-\mathrm{P}$ powder

Напыление покрытий проводили в ИТПМ CO РАН на установке плазменного напыления порошковых материалов «Термоплазма 50-01» с кольцевым вводом порошка. В качестве плазмообразующего и транспортирующего газа использовали воздух, в качестве защитного - смесь воздуха и пропан-бутана. Режимы плазменного напыления: сила тока - 180 А, напряжение 265 В, дистанция напыления - 170 мм. Порошок напыляли на трубы из низкоуглеродистой стали 20, внутренний диаметр которых был равен 25 мм, толщина стенки 3 мм. Непосредственно перед напылением поверхность труб подвергали пескоструйной обработке.

Структурные исследования выполняли с использованием оптического микроскопа Carl Zeiss Axio Observer A1m и растрового электронного микроскопа Carl Zeiss EVO 50 XVP с микроанализатором EDS X-Act. В качестве объектов выступали образцы, вырезанные из труб в направлении, перпендикулярном покрытию. Микрошлифы подготавливали по стандартной методике: механическое шлифование при помощи абразивных шкурок и полирование с использованием порошка оксида алюминия. Травление поверхности покрытий осуществляли раствором 10 мл $\mathrm{HCl}, 0,1$ мл $\mathrm{HNO}_{3}$ и 10 г $\mathrm{FeCl}_{3}$. Тонкое строение структуры полученных покрытий исследовали при помощи просвечивающего электронного микроскопа Technai G2 FEI. Образцы для просвечивающей электронной микроскопии готовили следующим образом: из покрытий вырезали заготовки диаметром 3 мм, которые механически утоняли до толщины 90...100 мкм при помощи абразивной бумаги. На механически 
утоненных заготовках вышлифовывали сферические лунки с использованием абразивных паст зернистостью от 5 до 1 мкм и суспензии оксида алюминия зернистостью 0,05 мкм. Ионное утонение полученных углублений фольг выполняли на установке Gatan PIPS model 659. Фазовый состав порошка и покрытий был изучен с использованием рентгеновского дифрактометра ARL

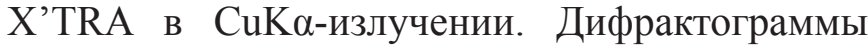
регистрировали в режиме времени $t=3 \mathrm{c}$ с шагом $\Delta 2 \theta=0,05^{\circ}$. Объемную долю частиц карбида бора в покрытиях оценивали при помощи программного обеспечения Axio Vision Multiphase (Carl Zeiss). Микротвердость структурных составляющих определяли на приборе Wolpert Group 402MVD при нагрузке 50 г.

\section{Результаты и обсуждение}

Фазовый состав частиц исходного порошка и покрытий приведен на рис. 2. Видно, что основными фазами порошкового материала являются карбид бора $\left(\mathrm{B}_{4} \mathrm{C}\right)$ и $\mathrm{Ni}$ (рис. $\left.2, a\right)$. Уширение пиков никеля на рентгенограмме порошка свидетельствует об его аморфном состоянии. Кроме того, наблюдаются рефлексы оксида бора $\left(\mathrm{B}_{2} \mathrm{O}_{3}\right)$. На рентгенограмме покрытий (рис. 2, б) присутствуют рефлексы боридов никеля $\left(\mathrm{Ni}_{3} \mathrm{~B}\right.$, $\mathrm{NiB}$ и $\left.\mathrm{Ni}_{4} \mathrm{~B}_{3}\right)$, $\mathrm{Ni}$ и карбида бора $\left(\mathrm{B}_{4} \mathrm{C}\right)$. Наличие оксидов никеля $(\mathrm{NiO})$ и бора $\left(\mathrm{B}_{2} \mathrm{O}_{3}\right)$ может сви-

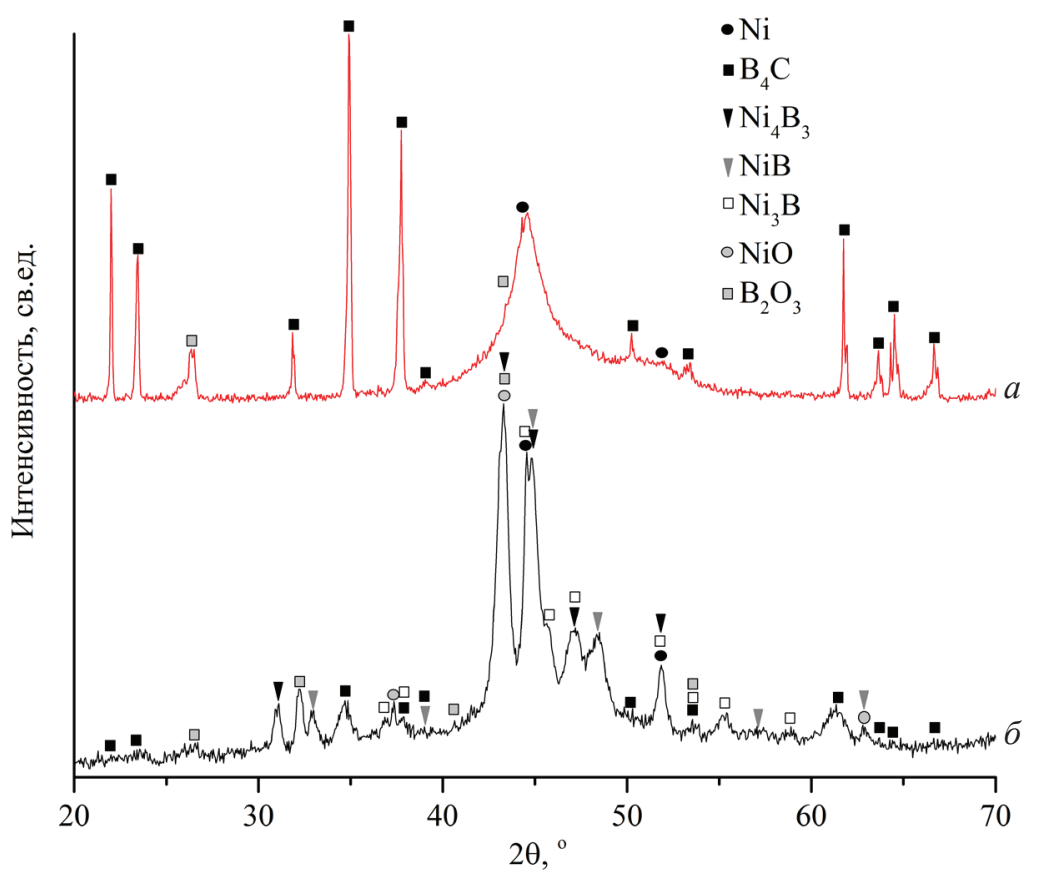

Puc. 2. Рентгенограммы порошка (a) и покрытия (б)

Fig. 2. XRD patterns of the powder $(a)$ and coating (б) детельствовать о недостаточной защите порошка в плазменной струе.

Изображение поперечного сечения покрытий представлено на рис. 3. Видно, что плазменное напыление композиционного порошка $\mathrm{B}_{4} \mathrm{C}-\mathrm{Ni}$-P позволяет сформировать плотные покрытия (пористость $\sim 1 \%$ ) толщиной около 1200 мкм. Граница раздела «покрытие-основной металл» выявляется в виде тонкой линии, отслоений не наблюдается (рис. 3, $a$ ).

Более детальное изображение материала покрытий приведено на рис. 3, б-г. Отчетливо видно, что темно-серые включения произвольной формы размерами до 30 мкм (рис. 3, б) равномерно распределены в металлической матрице. Согласно данным микрорентгеноспектрального анализа (таблица, участок 1) указанные включения являются нерасплавившимися до конца в плазменной струе частицами $\mathrm{B}_{4} \mathrm{C}$. Объемная доля карбида бора в покрытиях - около 24 \%.

Карбид бора разлагается по реакции: $\mathrm{B}_{4} \mathrm{C} \rightarrow 4 \mathrm{~B}(\mathrm{~TB})+\mathrm{C}(\mathrm{тв})$. Высокие скорости перемешивания и охлаждения материала покрытий способствуют неравномерному распределению химических элементов в металлической матрице, о чем свидетельствуют результаты микрорентгеноспектрального анализа (таблица, участки 2-4). Согласно полученным данным в результате взаимодействия $\mathrm{Ni}$ и $\mathrm{B}$ в материале покрытий формируются бориды никеля с различным химическим составом.

При избытке кислорода карбид бора окисляется по реакции $\mathrm{B}_{4} \mathrm{C}+4 \mathrm{O}_{2} \rightarrow \mathrm{CO}_{2}\left(\right.$ г) $+2 \mathrm{~B}_{2} \mathrm{O}_{3}$ (тв). Оксидная пленка $\mathrm{B}_{2} \mathrm{O}_{3}$, образующаяся на поверхности частиц $\mathrm{B}_{4} \mathrm{C}$, остается в покрытии вместе с карбидным ядром. В свою очередь, никель окисляется по реакции $2 \mathrm{Ni}+\mathrm{O}_{2} \rightarrow 2 \mathrm{NiO}(\mathrm{тв})$. Образовавшиеся оксиды остаются в металлической матрице покрытий (отмечены стрелками на рис. 3,2 ).

В результате травления поверхности покрытий было выявлено, что рядом с частицами $\mathrm{B}_{4} \mathrm{C}$ располагаются области с округлыми включениями размерами менее 500 нм (рис. 3, в, 2, область 5), химический состав которых указан в таблице (участок 5). Повышенное содержание $\mathrm{Ni}$, Р и В в этих 

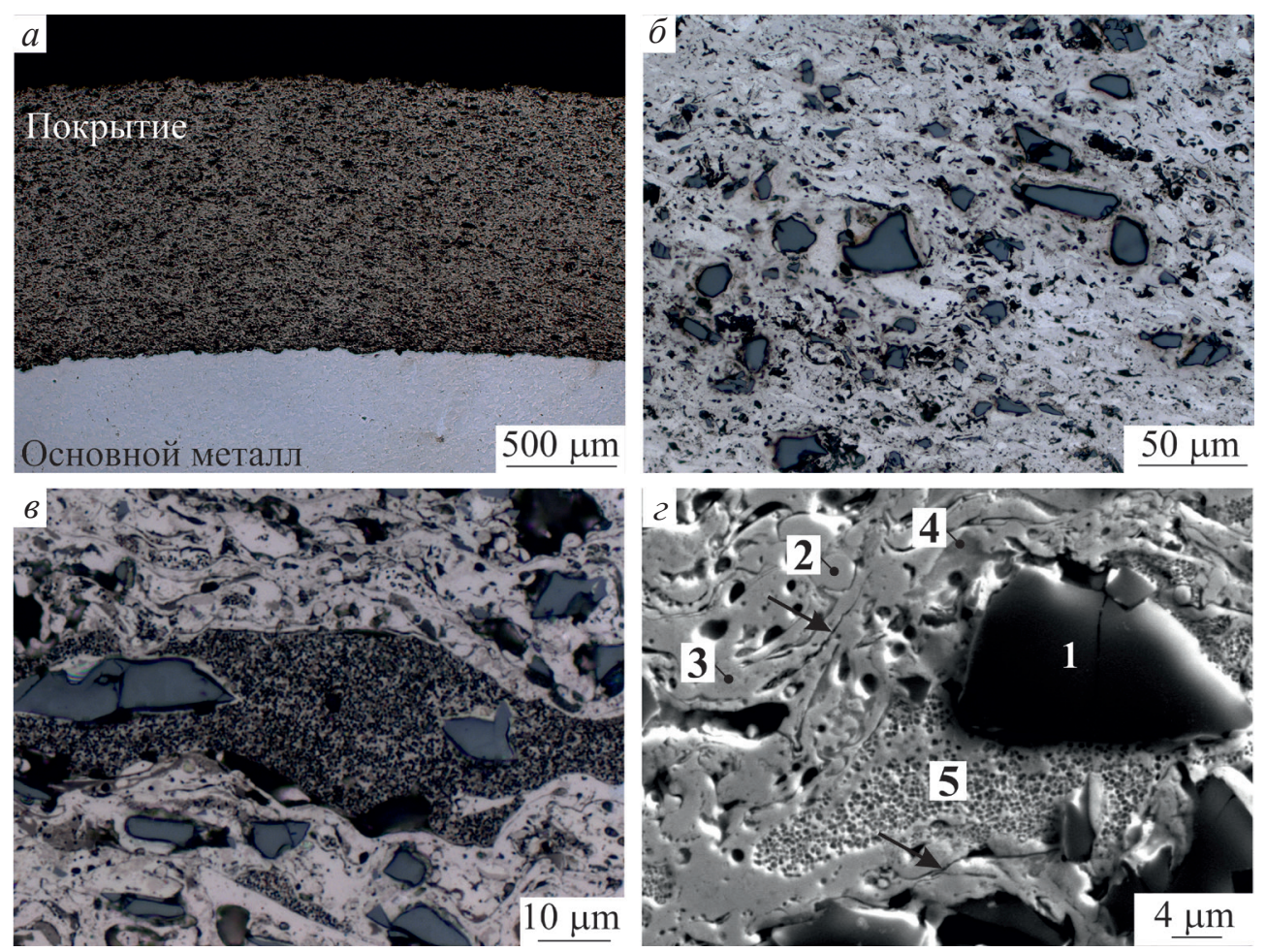

Puc. 3. Общий вид композиции «основной металл-покрытие» $(a)$; структура материала покрытия до травления (б); структура материала покрытия после травления $(8,2) ; a-8$ оптическая микроскопия; 2 - растровая электронная микроскопия в обратно-отраженных электронах:

1 - частица $\mathrm{B}_{4} \mathrm{C} ; 2$ - 4 - бориды никеля; 5 - эвтектические области. Стрелками отмечены оксиды никеля

Fig. 3. The image of «base metal-coating» compounds $(a)$; the material structure of the coating before etching $(\sigma)$; the material structure of the coating after etching $(b, 2) ; a-b-$ optical microscopy; 2 - scanning electron microscopy in the regime of reflected electrons:

$1-\mathrm{B}_{4} \mathrm{C}$ particle; $2-4$ - nickel borides; 5 - eutectic areas. The arrows show the nickel oxides

\section{Данные микрорентгеноспектрального анализа различных участков покрытия}

The data of micro-X-ray spectral analysis of various areas of coating

\begin{tabular}{|c|c|c|c|c|c|}
\hline \multirow{2}{*}{$\begin{array}{c}\text { Номер участка / } \\
\text { No. area }\end{array}$} & \multicolumn{5}{|c|}{$\begin{array}{c}\text { Химический элемент, ат. \% / } \\
\text { Chemical element, at. \% }\end{array}$} \\
\cline { 2 - 6 } & $\mathrm{Ni}$ & $\mathrm{B}$ & $\mathrm{C}$ & $\mathrm{O}$ & $\mathrm{P}$ \\
\hline 1 & 0,08 & 86,55 & 13,03 & 0,3 & 0,02 \\
\hline 2 & 57,71 & 22,73 & 8,22 & 2,12 & 9,22 \\
\hline 3 & 40,37 & 44,53 & 10,23 & 2 & 2,87 \\
\hline 4 & 49,65 & 25,69 & 13,10 & 3,23 & 8,33 \\
\hline 5 & 44,26 & 26,06 & 7,35 & 9,36 & 12,97 \\
\hline
\end{tabular}

областях свидетельствует о том, что основные реакции происходят с участием этих элементов.

Полученные данные подтверждаются дюрометрическими исследованиями. В результате локальных измерений микротвердости было установлено, что микротвердость частиц карбида бора находится в пределах $30 . . .40$ ГПа, металли- ческой матрицы - 4,0...5,5 ГПа, эвтектических участков - 2 ГПа.

Как было показано выше, высокие скорости охлаждения материала покрытий приводят к формированию структуры, идентификация которой методами оптической микроскопии является достаточно затруднительной. Тонкое стро- 
ение покрытий $\mathrm{B}_{4} \mathrm{C}-\mathrm{Ni}-\mathrm{P}$ изучали при помощи просвечивающей электронной микроскопии. На рис. 4 представлено изображение различных участков плазменных покрытий. В результате анализа полученных изображений было выявлено, что керамические частицы $\mathrm{B}_{4} \mathrm{C}$ окружены тонкими прослойками шириной до 1 мкм (рис. 4, a). Присутствие этих прослоек свидетельствует о надежном сцеплении частиц карбида бора с металлической матрицей. Более детальный анализ позволил выявить, что характерной особенностью материала прослоек является наличие округлых участков с кристаллической структурой размерами менее 10 мкм, расположенных в аморфной матрице (рис. 4,6$)$.
Анализ электронограммы, полученной при исследовании материала прослойки, подтверждает наличие аморфно-кристаллической структуры: хорошо видно широкое диффузное кольцо, а также точечные рефлексы от кристаллических фаз.

Участки, скорость охлаждения которых была ниже, характеризуются нанокристаллической структурой, что подтверждается соответствующей электронограммой (рис. 4, в). Видно, что размеры зерен составляют менее 20 нм. Различный контраст между зернами указывает на то, что они имеют разные кристаллографические ориентации. На рис. 4, 2 приведено изображение структуры, сформированной в условиях одновременного воздействия высоких степеней пере-

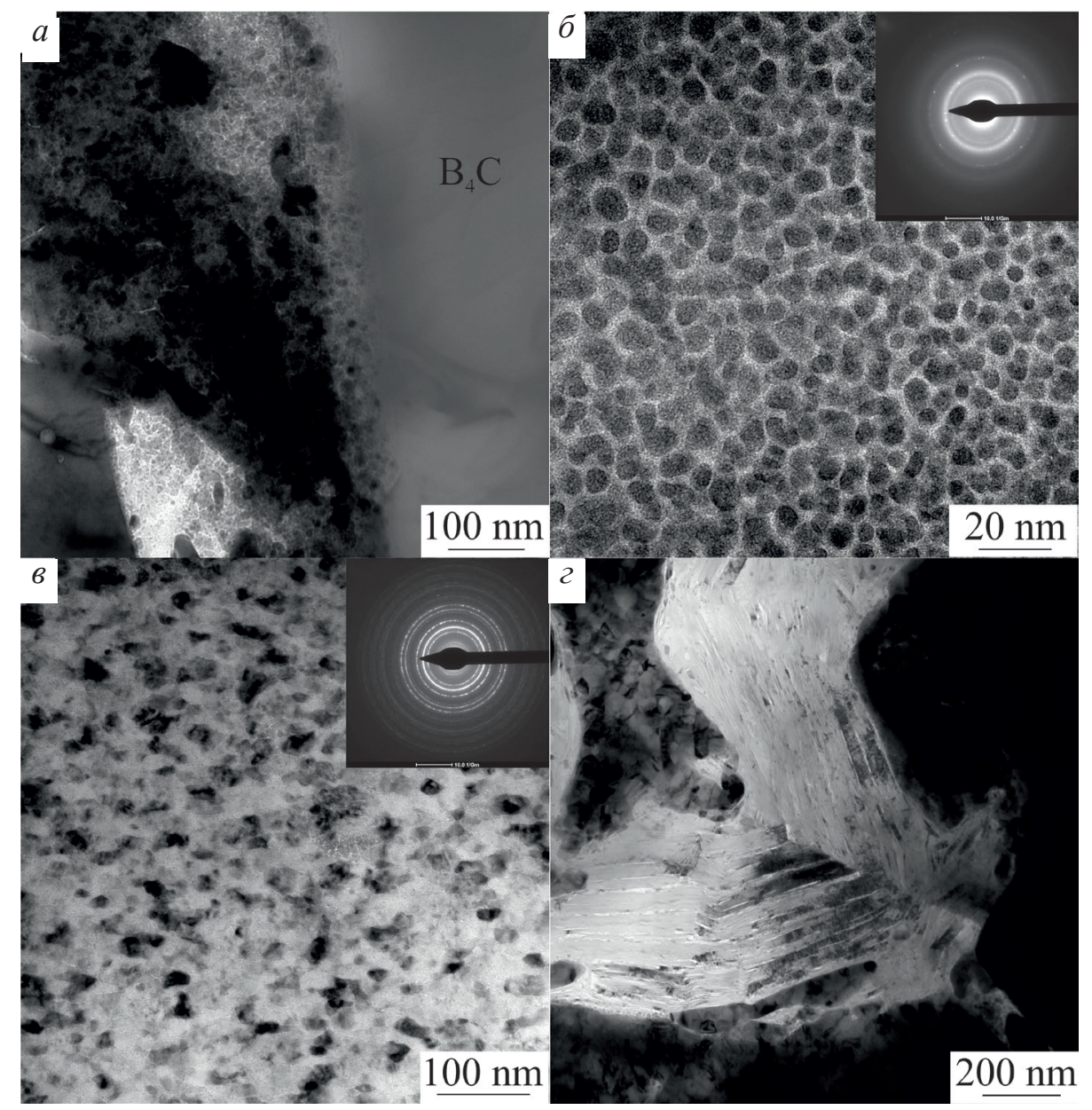

Puc. 4. Особенности тонкой структуры плазменных покрытий $\mathrm{B}_{4} \mathrm{C}-\mathrm{Ni}-\mathrm{P}$ : $a$ - частица $\mathrm{B}_{4} \mathrm{C}$ и прослойка; $\sigma$ - светлопольное изображение и дифракционная картина материала прослойки; 8 - светлопольное изображение и дифракционная картина наноструктурированного участка металлической матрицы; 2 - светлопольное изображение столбчатой структуры

Fig. 4. The feature of fine structure of $\mathrm{B}_{4} \mathrm{C}-\mathrm{Ni}-\mathrm{P}$ plasma coatings: $a-\mathrm{B}_{4} \mathrm{C}$ particle and interlayer; $\sigma$ - bright field image and electron diffraction of interlayer; 8 - bright field image and electron diffraction of metal matrix; 2 - bright field image of columnar structure 
охлаждения и пластической деформации. Видны пакеты столбчатых кристаллов шириной от 20 нм, которые растут от границ зерен. В свою очередь, каждый столбчатый кристалл состоит из более мелких выделений. Анализ строения металлической матрицы позволил выявить, что участки с нанокристаллической структурой и столбчатыми кристаллами располагаются в объеме покрытий случайным образом.

\section{Выводы}

1. Оптической микроскопией показано, что при помощи плазменного напыления удается сформировать качественные композиционные покрытия, характеризующиеся высокой плотностью.

2. Методами оптической и растровой электронной микроскопии, а также рентгенофазового анализа выявлено, что материал плазменных покрытий состоит из частиц $\mathrm{B}_{4} \mathrm{C}$ размерами до 30 мкм, равномерно распределенных в металлической матрице, представляющей собой бориды никеля $\left(\mathrm{Ni}_{3} \mathrm{~B}, \mathrm{NiB}\right.$ и $\left.\mathrm{Ni}_{3} \mathrm{~B}_{4}\right)$. Частицы карбида бора окружены областями с округлыми включениями. Данные области характеризуются повышенным содержанием никеля, фосфора и бора. В покрытии также присутствуют оксиды бора и никеля.

3. В результате локальных измерений микротвердости было установлено, что микротвердость частиц карбида бора находится в пределах 30...40 ГПа, металлической матрицы - 4,0 . .5,5 ГПа, областей с округлыми включениями - 2 ГПа.

4. Методом просвечивающей электронной микроскопии выявлено, что в процессе плазменного напыления между частицами карбида бора и металлической матрицей формируются тонкие прослойки, имеющие аморфно-кристаллическое строение, ширина которых не превышает 1 мкм. Металлическая матрица представляет собой участки с нанокристаллической структурой и столбчатыми кристаллами.

\section{Список литературы}

1. Бор в ядерной технике / В.Д. Рисованный, А.В. Захаров, Е.П. Клочков, Т.М. Гусева. - Димитровград: ГНЦ НИИАР, 2011. - 668 с. - ISBN 5-9483016-7.
2. Свойства, получение и применение тугоплавких соединений / под ред. Т.Я. Косолаповой. - М.: Металлургия, 1986. - 928 с.

3. Fabrication and tribological evaluation of vacuum plasma-sprayed $\mathrm{B}_{4} \mathrm{C} / \mathrm{H}$. Zhu, Y. Niu, C. Lin, L. Huang, H. Ji, X. Zheng // Coating Journal of Thermal Spray Technology. - 2012. - Vol. 21, iss. 6. - P. 1216-1223. doi: $10.1007 / \mathrm{s} 11666-012-9815-5$.

4. Wear of boron carbide ceramics by abrasive waterjets / K.A. Schwetz, L.S. Sigl, J. Greim, H. Knoch // Wear. - 1995. - Vol. 181-183. - P. 148155. - doi: 10.1016/j.wear.2005.01.020.

5. Al-Si/B ${ }_{4} \mathrm{C}$ composite coatings on Al-Si substrate by plasma spray technique / O. Sarikaya, S. Anik, S. Aslanlar, S.C. Okumus, E. Celik // Materials and Design. - 2007. - Vol. 28, iss. 9. - P. 2443-2449. doi: 10.1016/j.matdes.2006.09.007.

6. $\mathrm{B}_{4} \mathrm{C} / \mathrm{Ni}$ Composite coatings prepared by cold spray of blended or CVD-coated powders / C. Feng, V. Guipont, M. Jeandin, O. Amsellem, F. Pauchet, R. Saenger, S. Bucher, C. Iacob // Journal of Thermal Spray Technology. - 2012. - Vol. 21, iss. 3-4. - P. 561570. - doi: 10.1007/s11666-012-9774-x.

7. Effects of boron carbide content on the microstructure and properties of atmospheric plasmasprayed $\mathrm{NiCoCrAlY} / \mathrm{Al}_{2} \mathrm{O}_{3}-\mathrm{B}_{4} \mathrm{C}$ composite coatings / Y. Cao, C. Huang, W. Liu, W. Zhang, L. Du // Journal of Thermal Spray Technology. - 2014. - Vol. 23, iss. 4. P. 716-724. - doi: 10.1007/s11666-014-0061-x.

8. Zeng Y., Lee S.W., Ding C. Study on plasma sprayed boron carbide coating // Journal of Thermal Spray Technology. - 2002. - Vol. 11, iss. 1. - P. 129133. - doi: 10.1361/105996302770349069.

9. Zeng Y., Feng J., Ding C. Microstructure and properties of plasma spraing boron carbide coating // Journal of Materials Science and Technology. - 2000. Vol. 16, iss. 1. - P. 63-66.

10. Moradi M., Moazeni M., Salimijazi H.R. Microstructural characterization and failure mechanism of vacuum plasma sprayed Ti-6Al-4V/ $\mathrm{B}_{4} \mathrm{C}$ composite // Vacuum. - 2014. - Vol. 107. - P. 34-40. - doi: 10.1016/j. vacuum.2014.03.028.

11. Microstructure of vacuum plasma-sprayed boron carbide / H.R. Salimijazi, T.W. Coyle, J. Mostaghimi, L. Leblanc // Journal of Thermal Spray Technology. - 2005. - Vol. 14, iss. 3. - P. 362-368. doi: $10.1361 / 105996305 \times 59431$.

12. Microstructures and tribological properties of vacuum plasma sprayed $\mathrm{B}_{4} \mathrm{C}-\mathrm{Ni}$ composite coatings / H. Zhu, Y. Niu, C. Lin, L. Huang, H. Ji, X. Zheng // Ceramics International. - 2013. - Vol. 39, iss. 1. P. 101-110. - doi: 10.1016/j.ceramint.2012.05.101.

13. Preparation and oxidation behavior of $\mathrm{B}_{4} \mathrm{C}-\mathrm{Ni}$ and $\mathrm{B}_{4} \mathrm{C}-\mathrm{TiB}_{2}-\mathrm{TiC}-\mathrm{Ni}$ composite coatings produced by 
an HVOF process / M. Rafiei, M. Salehi, M. Shamanian, A. Motallebzadeh // Ceramics International. - 2014. Vol. 40, iss. 8, pt. B. - P. 13599-13609. - doi: 10.1016/j. ceramint.2014.05.081.

14. Comparison of the coatings deposited using $\mathrm{Ti}$ and $\mathrm{B}_{4} \mathrm{C}$ powder by reactive plasma spraying in air and low pressure / Z. Mao, J. Ma, J. Wang, B. Sun // Journal of Materials Science. - 2009. - Vol. 44, iss. 12. P. 3265-3272. - doi: 10.1007/s10853-009-3438-3.

15. Lin C., Kong M., Zhu H. Tribological behavior of vacuum plasma sprayed $\mathrm{B}_{4} \mathrm{C}$-Mo composite coating // Journal of Inorganic Materials. - 2016. - Vol. 31, iss. 1. P. 100-106. - doi: 10.15541/jim20150384.

16. Rafiei M., Salehi M., Shamanian M. Formation mechanism of $\mathrm{B}_{4} \mathrm{C}-\mathrm{TiB}_{2}-\mathrm{TiC}$ ceramic composite produced by mechanical alloying of $\mathrm{Ti}-\mathrm{B}_{4} \mathrm{C}$ powders // Advanced Powder Technology. - 2014. - Vol. 25, iss. 6. P. 1754-1760. - doi: 10.1016/j.apt.2014.07.003.

17. Ebrahimian-Hoaaeinabadi M., AzariDorcheh K., Moomir-Vaghefi S.M. Wear behavior of electroless Ni-P-B ${ }_{4} \mathrm{C}$ composite coatings // Wear. -
2006. - Vol. 260, iss. 1-2. - P. 123-127. - doi: 10.1016/j. wear.2005.01.020.

18. Влияние температуры оплавления на структуру и свойства самофлюсующихся покрытий на основе никеля / Е.Е. Корниенко, А.А. Никулина, А.Г. Баннов, В.И. Кузьмин, М. Мильдебрах, В.А. Безрукова, А.А. Жойдик // Обработка металлов (технология, оборудование, инструменты). - 2016. - № 4 (73). С. 52-62. - doi: 10.17212/1994-6309-2016-4-52-62.

19. Структура и свойства материалов из алюминидов никеля, полученных с использованием различных технологий / Е.Е. Корниенко, Л.И. Шевцова, Н.С. Белоусова, А.А. Никулина, А.И. Смирнов, В.И. Кузьмин, О.А. Рубцова // Перспективные материалы. - 2017. - № 3. - С. 49-58.

20. Effect of plasma spraying regimes on structure and properties of $\mathrm{Ni}_{3} \mathrm{Al}$ coatings / E.E. Kornienko, D.O. Mul', O.A. Rubtsova, S.P. Vaschenko, V.I. Kuzmin, I.P. Gulyaev, D.V. Sergachev // Thermophysics and Aeromechanics. - 2016. - Vol. 23, iss. 6. - P. 919-927. doi: $10.1134 / \mathrm{S} 0869864316060147$.

\section{Конфликт интересов}

Авторы заявляют об отсутствии конфликта интересов.

(c) 2017 Авторы. Издательство Новосибирского государственного технического университета. Эта статья доступна по лицензии Creative Commons «Attribution» («Атрибуция») 4.0 Всемирная (https://creativecommons.org/licenses/by/4.0/) 


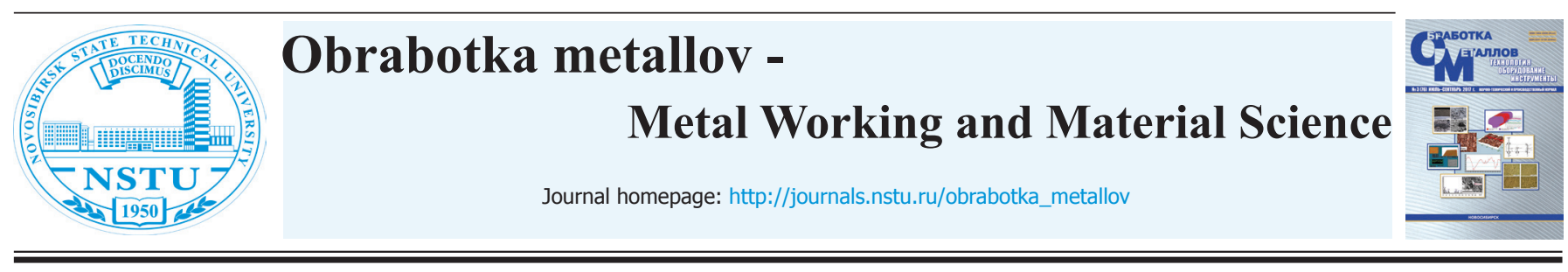

\title{
Structure Features of $\mathbf{B}_{4} \mathbf{C}-\mathrm{Ni}-\mathrm{P}$ Plasma Coatings
}

\author{
Elena Kornienko ${ }^{1, a, ",}$ Viktor Kuz'min ${ }^{2, b}$, Vasiliy Lozhkin ${ }^{1, c}$, Igor Gulyaev ${ }^{2, d}$, \\ Alexander Sivkov ${ }^{3,}$, Alexander Ivashutenko ${ }^{3, f}$, Ilyas Rahmatullin ${ }^{3, g}$, \\ Dmitrii Sergachev ${ }^{2, h}$, Viktoriya Bezrukova ${ }^{1, i}$
}

\footnotetext{
${ }^{1}$ Novosibirsk State Technical University, 20 Prospect K. Marksa, Novosibirsk, 630073, Russian Federation

${ }^{2}$ Khristianovich Institute of Theoretical and Applied Mechanics SB RAS, 4/1 Institutskaya str., Novosibirsk, 630090, Russian Federation

${ }^{3}$ National Research Tomsk Polytechnic University, 30 Lenin Avenue, Tomsk, 634050, Russian Federation

${ }^{a}$ (D) http://orcid.org/0000-0002-5874-5422, @ kornienko_ee@mail.ru, ${ }^{b}$ (D) http://orcid.org/0000-0002-9951-7821, @ vikuzmin57@mail.ru,

${ }^{c}$ (D) http://orcid.org/0000-0002-1686-0369, @ lozhkin@corp.nstu.ru, ${ }^{d}$ (D) http://orcid.org/0000-0001-5186-6793, @ gulyaev@itam.nsc.ru,

${ }^{e}$ (D) http://orcid.org/0000-0002-7685-5502, @ SivkovAA@mail.ru, ${ }^{f}$ (D http://orcid.org/0000-0002-6513-5438, @ ivaschutenko@mail.ru,

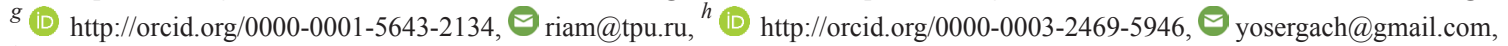

${ }^{i}$ (D) http://orcid.org/0000-0002-0329-9920, @ vikabezrukova1995@mail.ru
}

ARTICLE INFO

Article history:

Received: 28 June 2017

Revised: 28 July 2017

Accepted: 15 August 2017

Available online: 15 September 2017

Keywords:

Plasma spraying

Boron carbide

$\mathrm{B}_{4} \mathrm{C}-\mathrm{Ni}-\mathrm{P}$

Coating

\section{Acknowledgements.}

The research is carried out at Tomsk Polytechnic University within the framework of Tomsk Polytechnic University Competitiveness Enhancement Program grant.

\begin{abstract}
Purpose: This paper considers the structural features of $\mathrm{B}_{4} \mathrm{C}-\mathrm{Ni}-\mathrm{P}$ coatings obtained by air-plasma spraying with the unit for annular injection of powder. Materials and methods: $\left(\mathrm{B}_{4} \mathrm{C}\right)$ boron carbide powder clad with 30 wt. \% Ni-P is used. The powder is deposited on pipes of low-carbon steel $(0.2 \% \mathrm{C})$ by air-plasma spraying with the unit for annular injection of powder. The structure and phase composition of coatings are studied by optical microscopy, scanning electron microscopy with the microanalyser EDS, transmission electron microscopy and X-ray diffractometry. The microhardness measurements results are also presented. Results and discussion: The high-quality composite coatings with low porosity can be formed using plasma spraying. The combination of the optical and scanning electron microscopy as well as X-ray diffractometry revealed the composition of the plasma coatings. It consists of $\mathrm{B}_{4} \mathrm{C}$ particles up to $30 \mu \mathrm{m}$ sizes that are equally spaced in a metal matrix. The metal matrix consists of nickel borides $\left(\mathrm{Ni}_{3} \mathrm{~B}, \mathrm{NiB}\right.$ and $\left.\mathrm{Ni}_{3} \mathrm{~B}_{4}\right)$. Areas with roundish inclusions about $500 \mathrm{~nm}$ diameter are surrounded by boron carbide particles. These areas are characterized by a higher content of nickel, phosphorus and boron. $\mathrm{B}_{2} \mathrm{O}_{3}$ boron oxide and $\mathrm{NiO}$ nickel oxide are also presented in the coating. The microhardness local measurements demonstrated that the microhardness of boron carbide particles is $3000 \ldots 4000 \mathrm{HV}$, the metal matrix is $400 \ldots 550 \mathrm{HV}$ and the areas with roundish inclusions is $200 \mathrm{HV}$. According to the transmission electron microscopy data thin interlayers with an amorphous-crystalline structure up to $1 \mu \mathrm{m}$ width are formed between the boron carbide particles and the metal matrix. The metal matrix constitutes areas with a nanocrystalline structure and columnar crystals that located in the coatings volume at random.
\end{abstract}

For citation: Kornienko E.E., Kuz'min V.I., Lozhkin V.S., Gulyaev I.P., Sivkov A.A., Ivashutenko A.S., Rahmatullin I.A., D.V. Sergachev, Bezrukova V.A. Structure features of $\mathrm{B}_{4} \mathrm{C}-\mathrm{Ni}-\mathrm{P}$ plasma coatings. Obrabotka metallov (tekhnologiya, oborudovanie, instrumenty) $=$ Metal Working and Material Science, 2017. no. 3 (76), pp. 42-50. doi: 10.17212/1994-6309-2017-3-42-50. (in Russian).

\section{References}

1. Risovannyi V.D., Zakharov A.V., Klochkov E.P., Guseva T.M. Bor v yadernoi tekhnike [Boron in nuclear engineering]. Dimitrovgrad, GNTs NIIAR Publ., 2011. 668 p. ISBN 5-9483-016-7.

2. Alekseev A.G., Bovkun G.A., Bolgar A.S., Borisova A.L., Brakhnova I.T., Vinitskii I.M., Gordienko S.P., Goryachev Yu.M., Dvorina L.A., Drozdova S.V., Koval'chenko M.S., Kosolapova T.Ya., Kulik O.P., Obolonchik V.A., Paderno Yu.B., Panasyuk A.D., Popova O.I., Rud' B.M., Serebryakova T.I., Timofeeva I.I., Fomenko V.S. Svoistva,

\footnotetext{
* Corresponding author

Kornienko Elena E., Ph.D. (Engineering), Associate Professor

Novosibirsk State Technical University,

20 Prospect K. Marksa, 630073, Novosibirsk, Russian Federation

Tel.: 8 (383) 346-11-71, e-mail: kornienko_ee@mail.ru
} 
poluchenie i primenenie tugoplavkikh soedinenii [Properties, production and using of refractory compounds]. Ed. by T.Ya. Kosolapova. Moscow, Metallurgiya Publ., 1986. 928 p.

3. Zhu H., Niu Y., Lin C., Huang L., Ji H., Zheng X. Fabrication and tribological evaluation of vacuum plasmasprayed $\mathrm{B}_{4}$ C. Coating Journal of Thermal Spray Technology, 2012, vol. 21, iss. 6, pp. 1216-1223. doi: 10.1007/ s11666-012-9815-5.

4. Schwetz K.A., Sigl L.S., Greim J., Knoch H. Wear of boron carbide ceramics by abrasive waterjets. Wear, 1995, vol. 181-183, pp. 148-155. doi: 10.1016/j.wear.2005.01.020.

5. Sarikaya O., Anik S., Aslanlar S., Okumus S.C., Celik E. Al-Si/B ${ }_{4}$ composite coatings on Al-Si substrate by plasma spray technique. Materials and Design, 2007, vol. 28, iss. 9, pp. 2443-2449. doi: 10.1016/j.matdes.2006.09.007.

6. Feng C., Guipont V., Jeandin M., Amsellem O., Pauchet F., Saenger R., Bucher S., Iacob C. B C/Ni Composite coatings prepared by cold spray of blended or CVD-coated powders. Journal of Thermal Spray Technology, 2012, vol. 21, iss. 3-4, pp. 561-570. doi: 10.1007/s11666-012-9774-x.

7. Cao Y., Huang C., Liu W., Zhang W., Du L. Effects of boron carbide content on the microstructure and properties of atmospheric plasma-sprayed NiCoCrAlY $/ \mathrm{Al}_{2} \mathrm{O}_{3}-\mathrm{B}_{4} \mathrm{C}$ composite coatings. Journal of Thermal Spray Technology, 2014, vol. 23, iss. 4, pp. 716-724. doi: 10.1007/s11666-014-0061-x.

8. Zeng Y., Lee S.W., Ding C. Study on plasma sprayed boron carbide coating. Journal of Thermal Spray Technology, 2002, vol. 11, iss. 1, pp. 129-133. doi: 10.1361/105996302770349069.

9. Zeng Y., Feng J., Ding C. Microstructure and properties of plasma spraing boron carbide coating. Journal of Materials Science and Technology, 2000, vol. 16, iss. 1, pp. 63-66.

10. Moradi M., Moazeni M., Salimijazi H.R. Microstructural characterization and failure mechanism of vacuum plasma sprayed Ti-6Al-4V/B ${ }_{4}$ C composite. Vacuum, 2014, vol. 107, pp. 34-40. doi: 10.1016/j.vacuum.2014.03.028.

11. Salimijazi H.R., Coyle T.W., Mostaghimi J., Leblanc L. Microstructure of vacuum plasma-sprayed boron carbide. Journal of Thermal Spray Technology, 2005, vol. 14, iss. 3, pp. 362-368. doi: 10.1361/105996305X59431.

12. Zhu H., Niu Y., Lin C., Huang L., Ji H., Zheng X. Microstructures and tribological properties of vacuum plasma sprayed $\mathrm{B}_{4} \mathrm{C}-\mathrm{Ni}$ composite coatings. Ceramics International, 2013, vol. 39, iss. 1, pp. 101-110. doi: 10.1016/j. ceramint. 2012.05.101.

13. Rafiei M., Salehi M., Shamanian M., Motallebzadeh A. Preparation and oxidation behavior of $B_{4} C-N i$ and $\mathrm{B}_{4} \mathrm{C}-\mathrm{TiB}_{2}$-TiC-Ni composite coatings produced by an HVOF process. Ceramics International, 2014, vol. 40, iss. 8, pt. B, pp. 13599-13609. doi: 10.1016/j.ceramint.2014.05.081.

14. Mao Z., Ma J., Wang J., Sun B. Comparison of the coatings deposited using Ti and $\mathrm{B}_{4} \mathrm{C}$ powder by reactive plasma spraying in air and low pressure. Journal of Materials Science, 2009, vol. 44, iss. 12, pp. 3265-3272. doi: 10.1007/s10853-009-3438-3.

15. Lin C., Kong M., Zhu H. Tribological behavior of vacuum plasma sprayed $\mathrm{B}_{4} \mathrm{C}-\mathrm{Mo}$ composite coating. Journal of Inorganic Materials, 2016, vol. 31, iss. 1, pp. 100-106. doi: 10.15541/jim20150384.

16. Rafiei M., Salehi M., Shamanian M. Formation mechanism of $\mathrm{B}_{4} \mathrm{C}-\mathrm{TiB}_{2}-\mathrm{TiC}$ ceramic composite produced by mechanical alloying of Ti-B $\mathrm{B}_{4} \mathrm{C}$ powders. Advanced Powder Technology, 2014, vol. 25, iss. 6, pp. 1754-1760. doi: 10.1016/j.apt.2014.07.003.

17. Ebrahimian-Hoaaeinabadi M., Azari-Dorcheh K., Moomir-Vaghefi S.M. Wear behavior of electroless Ni-P$\mathrm{B}_{4} \mathrm{C}$ composite coatings. Wear, 2006, vol. 260, iss. 1-2, pp. 123-127. doi: 10.1016/j.wear.2005.01.020.

18. Kornienko E.E., Nikulina A.A., Bannov A.G., Kuz’min V.I., Mildebrath M., Bezrukova V.A., Zhoidik A.A. Vliyanie temperatury oplavleniya na strukturu i svoistva samoflyusuyushchikhsya pokrytii na osnove nikelya [The influence of flowing temperature on the structure and properties of the self-fluxing coatings]. Obrabotka metallov (tekhnologiya, oborudovanie, instrumenty) $=$ Metal Working and Material Science, 2016, no. 4 (73), pp. 52-62. doi: 10.17212/1994-6309-2016-4-52-62.

19. Kornienko E., Shevtsova L., Belousova N., Nikulina A., Smirnov A., Kuz'min V., Rubtsova O. Struktura i svoistva materialov iz alyuminidov nikelya, poluchennykh $\mathrm{s}$ ispol'zovaniem razlichnykh tekhnologii [Structure and properties of the nickel aluminide materials obtained using different technologies]. Perspektivnye materialy $=$ Journal of Advanced Materials, 2017, no. 3, pp. 49-58.

20. Kornienko E.E., Mul' D.O., Rubtsova O.A., Vaschenko S.P., Kuzmin V.I., Gulyaev I.P., Sergachev D.V. Effect of plasma spraying regimes on structure and properties of $\mathrm{Ni}_{3} \mathrm{Al}$ coatings. Thermophysics and Aeromechanics, 2016, vol. 23, iss. 6, pp. 919-927. doi: 10.1134/S0869864316060147.

\section{Conflicts of Interest}

The author declare no conflict of interest.

(C) 2017 The Authors. Published by Novosibirsk State Technical University. This is an open access article under the CC BY license (http://creativecommons.org/licenses/by/4.0/). 